\title{
Applications for investigating therapy progress of autistic children
}

\author{
Agata Kołakowska, Agnieszka Landowska, Michal R. Wrobel \\ Faculty of Electronics, Telecommunications and Informatics, \\ Gdansk University of Technology, Poland \\ Email: \{agatakol,nailie,wrobel\}@eti.pg.gda.pl
}

\author{
Dominika Zaremba, Dominika Czajak, \\ Anna Anzulewicz \\ Harimata Sp. z o.o., Poland \\ Email: \{dominika.zaremba,domnika,ania\}@harimata.co
}

\begin{abstract}
The paper regards supporting behavioral therapy of autistic children with mobile applications, specifically applied for measuring the child's progress. A family of five applications is presented, that was developed as an investigation tool within the project aimed at automation of therapy progress monitoring. The applications were already tested with children with autism spectrum disorder. Hereby we analyse children' experience with the games, as a positive attitude towards the application is the key factor enabling practical application of the solutions in therapy. The study might be interesting for both researchers and practitioners applying e-technologies in autistics therapy.
\end{abstract}

\section{INTRODUCTION}

A UTISM is a developmental disorder, that influences the ability to socialize, communicate as well as learning skills. Autistic children have diverse level of deficits in language understanding, speaking and other areas, that make the therapy and education very difficult [1]. Educating children with autism spectrum disorder could be a challenge in the best of circumstances [2]. There are premises for supporting therapy with e-technologies [3], [4], as most of the autistic children are eagerly using computers and tablets once they are taught how to use them [2]. Autistics require repetitive environment for functioning and learning. Technologies are able to perform the same activities in exactly the same way and with indefinite patience. Moreover, systems and devices might be customized in order to adjust to a unique set of deficits of an individual [5].

This study is performed under the AUTMON (Automated therapy monitoring for children with autism spectrum disorder) project, that aims at development of methods and tools to allow for the automatic evaluation of the therapy progress among children with autism spectrum disorder (ASD) [6]. Therapy monitoring is based on automatic detection of behavioral patterns in tablet and application usage. During the project 5 applications were developed or adjusted to fit the deficits of autistic children. The apps have the potential of being applicable in the therapy progress monitoring. However, the crucial question is: whether the autistic children are eager

This research is supported by the National Centre for Research and Development, Poland under grant AUTMON no IS-2/6/NCBR/2015, as well as DS Programs of the Faculty of Electronics, Telecommunications and Informatics, Gdansk University of Technology. This research is also inspired by European Cooperation in Science and Technology (COST) Action TD1309 Play for children with disabilities (LUDI). to use those games. This study aims at verification of this issue. In this paper, the applications and their adaptation to autistic users is described, and the data from interaction sessions is analyzed. Moreover, we report our observations on autistics interaction with tablets and applications. The study might be interesting for both producers of solutions for autistic children as well as for researchers investigating effective methods for ASD support and therapy with e-technologies.

The paper is organized as follows: Section II reports work related to this study and especially previous works on monitoring autistic users performance. Section III presents the study context and study design. Section IV and V present the results of games evaluation, and those sections are followed by a summary of results and discussion (Section VI) and the conclusions describing future works (Section VII).

\section{RELATED WORK}

New technologies are used while working with people suffering from autism both at the stage of diagnosis and therapy. The use of technology in this field is usually based on software implementations for common therapeutic tasks. Supporting diagnosis is limited to the use of computer versions of diagnostic questionnaires. Although there are some advanced technological solutions that can help to diagnose autism, they are still experimental methods, used in laboratories only or tested on small groups of people. In many cases they require specialized hardware. Some of these solutions are based on: eye tracking [7], automatic analysis of children video recordings [8], automatic analysis of individual hand movements [9] and machine learning algorithms applied to predict the state of a person [10].

Much more often than in the diagnosis, new technologies are used in the therapy of autism. In most cases, these solutions are computer implementations of paper-based therapeutic tools. There are numerous programs supporting daily activities, allowing for arranging and following procedures that consist of sequences of activities represented as images or text [5].

Numerous tools are created to help to acquire emotional intelligence skills. These include programs that help in learning face and emotion recognition, learning expressing emotions through facial expressions [11] and recognizing emotions of encountered people [12]. There are also solutions, that incorporate special hardware measuring physiological signals 
to interpret an emotional state of a person, which may be especially valuable information in case of autistic children, who often are not able to show their emotions [13], [14].

Apart from solutions designed for people with autism, there are also some applications supporting work of the therapists.

\section{STUDY CONTEXT AND STUDY DESIGN}

\section{A. Study context and purpose}

In 2015 a project named AUTMON was started by a consortium consisting of Gdansk University of Technology, Harimata LTD and Hippotherapy Foundation [6]. Its goal is to develop methods and tools for automated monitoring of progress in the therapy of children with autism.

The goal is addressed by (1) the development of technology identifying behavioral patterns associated with autism, which could be used to test and evaluate the progress of therapy and by (2) implementation of a system that automatically tracks these patterns during therapy. A specially designed tablet applications have been created to record the behavioral patterns during the childs interaction with tablet. These patterns, which may be analyzed for diagnostic or therapeutic purposes, contain information on: touch screen gestures, e.g. tap and swipe device movements identified by accelerometer; the application usage, i.e. navigationpatterns, objects that attract attention (goals/distractors), strategies of decision making etc. The research goal of the AUTMON project is to verify, that the proposed solution might support the therapists by providing them with objective data on the progress of therapy of autistic children in various areas of their development.

The first step in the project research is verification of the children' experience with the games, as attitude towards the application is the key factor enabling practical application of the solutions in therapy. Reporting the study of children experience with the games is the main goal of this paper.

The key research question addressed by the presented study might be formulated as follows: Which applications provide experiences positive enough for the child to perform and continue interaction allowing (as a result) to monitor progress.

\section{B. Applications under investigation}

The applications developed during the project were developed in the cooperative and iterative process engaging psychologists, therapists and data scientists. They were designed for the tablet devices. The solution set includes five games, which allows to gather information about children interactions with the device, using touch screen sensors and gyroscope.

Each game consists of training phase and actual game. During the training phase child may get acquainted with the game concept. At the beginning of training session interactive tutorial is presented. Later child may try to use application by herself/himself. During the whole training therapists should instruct the child and may use either verbal and physical guidelines. Depending on game, training session lasts from 2 to 5 minutes. At any time training session may be terminated by tapping three fingers at the top of the screen.
After finishing training session, actual game, which is used to collect data, begins. In order to obtain the undisturbed results, during this phase, the therapist should not interact with the child. Like during the training phase the session can be terminated, when a child looses interest in the game. Fig. 1 presents the games screenshots.

1) Boxes: Boxes is a game designed for warm-up. The goal is to place the balls in boxes by matching corresponding colors.

2) Sharing: The goal of the second game is to share food among four animated children. Child has to tap on the displayed food article (watermelon, apple, cake) and swipe portions to four plates in front of the children.

3) Cat \& Dog: Based on the experimental paradigm of Go/NoGo, the game is intended to be used by older children. Typically developing 3-4 years old's are not ready to proceed with this cognitively demanding task.

4) Pinwheel: The pinwheel is slowly turning around while a color ball balances at the base of its stem. A child has to flip the tablet (previously only touch screen data were gathered, now accelerometer and gyroscope provide the data) precisely to move the color ball to the corresponding "pinwheel petal".

5) Creativity: Drawing and coloring pictures seem to be the most rewarding game for most children.
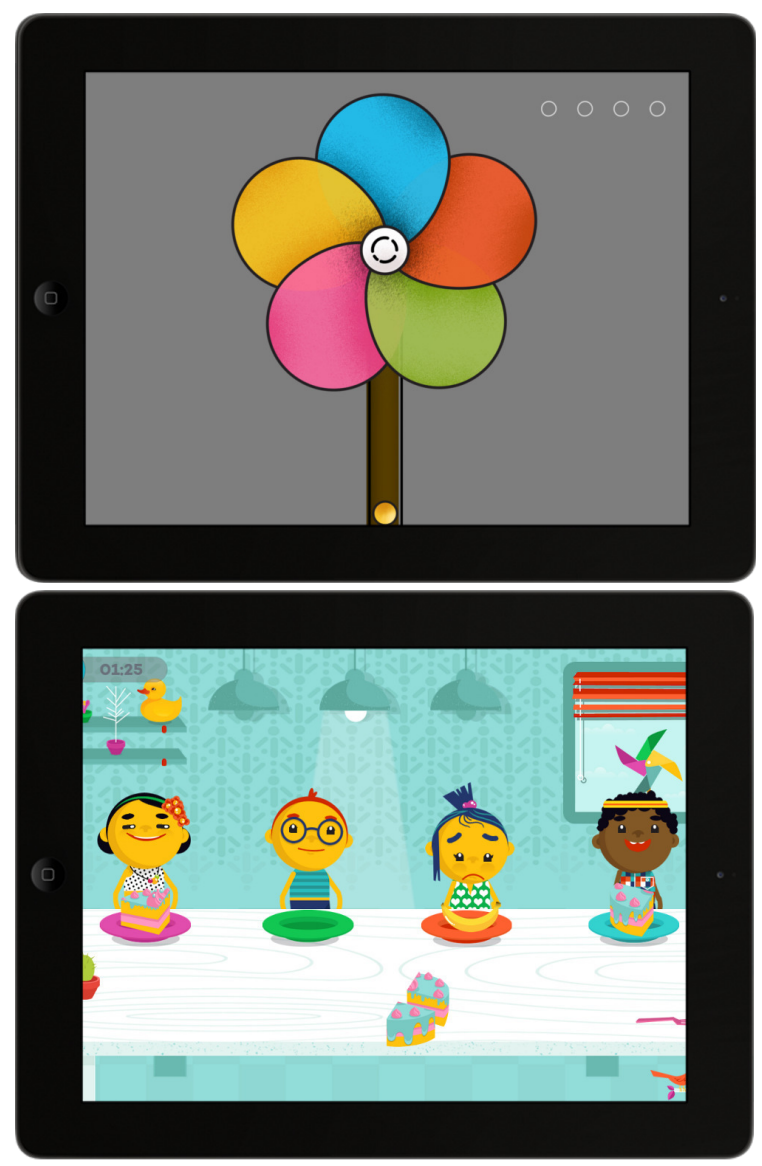

Fig. 1. Sample games screenshots 


\section{Study design}

Two evaluation methods were applied: (1) a behavioral study of video recordings of children interacting with the games and (2) on-line behavioral tagging during measurement sessions.

The first part of the study was performed before measurements sessions were started and aimed at evaluation of user experience with the games. The second part of the study is performed on-line during each measurement session and this paper summarizes the results as a validation of the chosen approach. The target group in those studies are children aged 3 up to 7 (kindergarten education level), diagnosed (or during diagnosis) with autism spectrum disorder. As children measurements require consent from parents, randomization of selection to sample was not possible. Children were recruited among pupils of ten therapeutic centers in Poland. Parents' agreement rate was very high (more than 90\%).

1) User experience study: This study was aimed at analyzing the experience of the children play. The first step of this study was to find a definition and unambiguous indicators of good UX. Previous research on the subject mentioned: engagement, playfulness and fun as indicators of good UX in children [15]. Previous studies [16] used some combinations of behavioral observation with survey and interview techniques. This approach was hard to apply in this study, because children with autism are mostly nonverbal or of poor communication skills, so we couldn't ask them straightforward for their opinions. Therefore in this study behavioral observation was chosen as an investigation tool. Moreover, the gameplay was recorded and analysis were made post-hoc by independent observers. This approach allowed for measuring consistency of manual tagging in order to achieve higher reliability. Steps of the study preparation:

1) An exploratory observation of gameplay was performed resulting in a list of observational indicators of interest and having fun with the games. Three independent judges (two psychologist with specialization in autism therapy and a naive observer not familiar with the symptoms of autism) were watching videos and listing observational indicators of good/bad experience of play.

2) Behaviors listed by observers in the previous step were clustered into three categories of: engagement, understanding and enjoyment.

3) The three factors were conceptualized and operationalized with three behavioral indicators each forming 9item behavioral observation scheme.

4) Each item was assigned a five-point discrete scale (1-5) with descriptive explanations assigned to first and last item. As a result an observation sheet was prepared, that was used in manual tagging of the video recordings.

5) Using the elaborated scale and observation sheet, manual tagging of videos was performed by 5 independent observers. Total number of 21 recordings were analyzed.

6) The manual markers from observers were checked against consistency criteria using commonly used Kappa coefficient. The data was used to evaluate the applications using the understanding, engagement and enjoyment criteria.

7) Final results were formulated regarding inclusion or exclusion of the games from the measurement procedure. Some additional recommendations were formulated for the measurement procedure.

Understanding factor characterizes, that the device functions and the games are understandable for a child. First of all, child realizes that tablet screen can be used to interact with the tablet. There is a range of algorithms of interaction (based on set of possible actions provided) that result in successful interaction with the game, meaning when child achieves the goal of the game. Child wins the game if (s)he acquires a final stage of task completion, e.g. feeds all four children, draws a picture, paints a picture etc.) Children may get to win by themselves or with a standarized verbal or motor prompt from the experimenter.

Engagement factor characterizes, that the function of the tablet and the game is engaging, meaning, that tablet and game attracts childs attention. If in childs sight, in proximity of his/her hands there is a possibility of engaging. It can be observed how often, regular and persistent is childs interaction with the touch screen.

Enjoyment factor characterizes, whether interaction with tablet results in growth of positive affect symptoms, which were operationalized as laughing and vocal expressions, verbal expressions, mimic expressions and motor and postural expressions (clapping hands, jumping, shrugging). Facial expressions were not observable due to to the fact, that video materials were filmed from the chin down (which was explicitly stated in the parent-experimenter agreement for the privacy reasons).

The results of the study are provided in Section IV.

2) Validation during measurement sessions: The second study is on-line observation made during the measurement sessions.

The children are supposed to play all five games during the sessions. The detailed criteria used in the usability study were significantly simplified for the measurement stage. From the 9-item scale only 2 indicators were used in the on-line tagging procedure. For each game the following two factors are estimated by an observer: the level of difficulty, which may be 1-easy, 2-adequate, 3-difficult; the level of interest, which may be 0 -none, 1-low, 2-average, 3-high. If a child does not want to play a game in spite of some encouragement, 0 is assigned to the level of interest and no value is given as the level of difficulty. If, for some reasons, a therapist decides not to try to play a game, neither difficulty nor interest are assigned a value. The reasons for childs intentional exclusion from play might fall into following categories: a child performed an extremely negative reaction to the game (eg. fear of sounds); a child is in bad disposition on this specific session day (regular therapist opinion); a child got upset during the study procedure.

The difficulty factor corresponds to understandability criteria from the UX study, the interest factor corresponds to engagement criteria from the UX study. 


\section{USABILITY EVALUATION OF APPLICATIONS FOR PROGRESS MONITORING}

This section provides results of usability evaluation of the applications. 21 recordings of nine children have been annotated. All children were boys, aged 3-7. Each of them played from one to four of the created games.

To evaluate the consistency of the annotations done by four observers, kappa coefficient was calculated. The values obtained for all nine behavior indicators defined in section III-C were averaged over all games. The highest agreement was obtained for engagement factors $(0.79 ; 0.70$ and 0.61 respectively), moderate for understanding factors $(0.57 ; 0.54$ and 0.43 ) and the lowest for enjoyment (0.40; 0.30 and 0.38$)$.

To provide final evaluation scores for the three categories (engagement, understanding, enjoyment) the following procedure has been followed independently for each game:

1) in each category the scores for three behavior indicators were summed getting total category scores;

2) the total category scores were averaged over four judges; as a result three values for engagement, understanding and enjoyment were obtained for each recording;

3) the obtained recording scores were averaged over all films presenting a given game.

The final results are presented in Table I. Sharing received the highest scores for understanding and engagement. Surprisingly, Cat \& Dog took the second place in these two categories and the first one in enjoyment. That game seemed to be rather difficult, some of the children did not play it at all. In spite of these obstacles, it turned out to be entertaining. Creativity game received the lowest scores for understanding and enjoyment, Pinwheel for engagement. Although Creativity seemed to be understandable for the children, as most of them intuitively drew lines with their fingers, precise drawing turned out to be quite difficult bringing about the low scores. It can be observed that more understandable games get higher scores in engagement and enjoyment, however the relationship between the variables would require further analysis.

\section{ON-LINE TAGGING - EXECUTION AND RESULTS}

To summarize the results the first three sessions of each child have been taken into account. Due to some absence rate not all participants have already gathered three data records. From the total number of 42 children, 29 of them were present during all three sessions. The absence rate is typical for the kindergarten children age range.

The results obtained for each game are averaged over all children. The final scores for the levels of difficulty and interest are shown in Table II.

It can be seen that three of the games (Sharing, Boxes, Creativity) turned out to be much more interesting than other two (Pinwheel, Cat and Dog). Some relation between interest and difficulty may be observed. The more difficult game, the less interesting and vice versa. Boxes and Sharing were the easiest ones. Pinwheel, which requires motor skills while flipping the tablet, and Cat \& Dog requiring being focused, were the most difficult, just as it was expected. Some of the kids were not able to play Cat \& Dog at all.

Another interesting observation is the fact that the levels of interest and difficulty do not change much over the time. It meant that data collected while playing are not affected by confounding factor such as history effect.

\section{SUMMARY OF RESULTS AND DISCUSSION}

The aim of this paper was to evaluate, whether the applications developed for monitoring progress of children with autism have a chance to be used in practice. The question was raised, whether the applications are engaging, understandable and enjoying enough to trigger and maintain childs focus and interaction.

The main results might be formulated as follows:

- understanding, engagement and enjoyment are partially dependent, eg. some children are fascinated with pinwheel although they do not understand the purpose of the game, for the others, if they do not understand a game, they are not interested in it.

- both studies confirmed, that three of the games: Sharing, Creativity and Boxes are mostly understandable for the children; out of the three, Sharing seems to be the favorite game;

- game Cats \& Dogs and Pinwheel seems less intuitive and half of the children refuse to use them, however, once a child understands the game, it seems to be engaging,

- although it seemed, that some of the children do remember the games after a month (average time distance between recording sessions), the data do not confirm the history effect, which is convenient for measurement.

All recording and measurement sessions revealed also some qualitative observations:

- Most of the children with autism were eager to use tablets, typical behavior was to grab a tablet in the proximity of hand and to follow it, when it was taken away,

- Most of the children know very well, how to turn the tablet on and how to switch to another game, we have used the parent mode in order to prevent children from switching the games off;

- Some lower functioning children got frustrated, when they did not understand the game;

- Some higher functioning children got bored easily and even though they started eagerly, it was hard to keep them play for longer than a minute;

Authors are aware of the fact, that this study is not free of some limitations, such as small number of videos analyzed and low consistency of manual tagging for some of the variables. Despite some limitations of the study, the performed analysis allowed us to determine, which applications provide experiences positive enough for the child to perform and continue interaction allowing (as a result) to monitor progress. The goal of the study was achieved. 
TABLE I

USER EXPERIENCE RESULTS OBTAINED FOR THE APPLICATIONS

\begin{tabular}{|l|c|c|c|c|c|c|c|c|c|c|c|c|}
\hline \multirow{2}{*}{ Game name } & \multicolumn{4}{|c|}{ Understanding } & \multicolumn{4}{c|}{ Engagement } & \multicolumn{4}{c|}{ Enjoyment } \\
\cline { 2 - 15 } & Min & Max & Avg & SD & Min & Max & Avg & SD & Min & Max & Avg & SD \\
\hline Boxes & 10 & 13 & nd & nd & 15 & 15 & nd & nd & 8 & 11 & nd & nd \\
\hline Sharing & 8 & 15 & 12,8 & 3,3 & 14 & 15 & 14,7 & 0,6 & 7 & 12 & 8,9 & 2,3 \\
\hline Pinwheel & 4 & 15 & 9,5 & 5,2 & 8 & 15 & 12,9 & 3,1 & 4 & 12 & 8,8 & 3,3 \\
\hline Creativity & 6 & 13 & 8,9 & 3 & 11 & 14 & 13,4 & 1,5 & 2 & 10 & 7,8 & 1,8 \\
\hline Cat \& Dog & 6 & 15 & 11,3 & 4,3 & 12 & 15 & 14,2 & 1,5 & 5 & 13 & 9,3 & 3,3 \\
\hline
\end{tabular}

TABLE II

INTEREST AND DIFFICULTY LEVELS FOR THREE MEASUREMENT SESSIONS

\begin{tabular}{|c|c|c|c|c|c|c|c|c|}
\hline Game name & \multicolumn{4}{|c|}{ Interest } & \multicolumn{4}{|c|}{ Difficulty } \\
\cline { 2 - 9 } & Total* & $\begin{array}{c}\text { 1st } \\
\text { session** }\end{array}$ & $\begin{array}{c}\text { 2nd } \\
\text { session** }\end{array}$ & $\begin{array}{c}\text { 3rd } \\
\text { session** }\end{array}$ & Total* & $\begin{array}{c}\text { 1st } \\
\text { session** }\end{array}$ & $\begin{array}{c}\text { 2nd } \\
\text { session** }\end{array}$ & $\begin{array}{c}\text { 3rd } \\
\text { session** }\end{array}$ \\
\hline Boxes & 2,5 & 2,4 & 2,4 & 2,5 & 1,9 & 1,8 & 1,9 & 1,9 \\
$\mathrm{n} *=100 \mathrm{n} * *=28$ & $(0,8)$ & $(0,8)$ & $(0,7)$ & $(0,8)$ & $(0,5)$ & $(0,6)$ & $(0,6)$ & $(0,5)$ \\
\hline Sharing & 2,6 & 2,7 & 2,7 & 2,6 & 1,8 & 1,9 & 1,9 & 1,7 \\
$\mathrm{n} *=104 \mathrm{n} * *=28$ & $(0,6)$ & $(0,5)$ & $(0,6)$ & $(0,5)$ & $(0,5)$ & $(0,5)$ & $(0,5)$ & $(0,5)$ \\
\hline Pinwheel & 1.9 & 2,1 & 1,8 & 2,1 & 2,6 & 2,6 & 2,6 & 2,4 \\
$\mathrm{n} *=85 \mathrm{n} * *=22$ & $(0,9)$ & $(0,9)$ & $(1,0)$ & $(0,8)$ & $(0,6)$ & $(0,5)$ & $(0,5)$ & $(0,7)$ \\
\hline Creativity & 2,4 & 2,6 & 2,5 & 2,4 & 2,3 & 2,2 & 2,4 & 2,2 \\
$\mathrm{n} *=102 \mathrm{n} * *=28$ & $(0,8)$ & $(0,7)$ & $(0,7)$ & $(0,7)$ & $(0,5)$ & $(0,5)$ & $(0,5)$ & $(0,5)$ \\
\hline Cat \& Dog & 1,8 & 1,9 & 1,9 & 1,9 & 2,7 & 2,6 & 2,8 & 2,7 \\
$\mathrm{n} *=49 \mathrm{n} * *=13$ & $(1,0)$ & $(0,9)$ & $(1,0)$ & $(1,0)$ & $(0,6)$ & $(0,8)$ & $(0,4)$ & $(0,5)$ \\
\hline
\end{tabular}

$\mathrm{n}^{*}$ - total no of gameplays evaluated (all children, up to 3 sessions)

$\mathrm{n}^{* *}$ - no of gameplays evaluated (only children with 3 sessions)

\section{CONCLUSIONS}

Our study shows the potential for use of tablet-based technology in children therapy. Children are generally enthusiastic about tablets and other mobile devices and this rapture can be utilized in the area of research, diagnosis and therapy. Future works in this discipline should focus on to developing tools for monitoring progress and better means for their evaluation. As our study concludes, its not easy to evaluate in terms of child experience, especially in autistic population, where raters agreement is hard to reach.

Objective measures of therapy progress can bring quality data to the therapy providers. As they have better knowledge of childs state and developmental pace and direction, thy can provide adjusted intervention to best fit the individual needs of the child. Better therapy means better life for children with ASD, as enormous amount of evidence shows that personalized and early onset intervention improve their future quality of life.

\section{REFERENCES}

[1] F. R. Volkmar, R. Paul, A. Klin, and D. J. Cohen, Handbook of Autism and Pervasive Developmental Disorders, Diagnosis, Development, Neurobiology, and Behavior. John Wiley \& Sons, 2005, vol. 1.

[2] L. Winerman, "Effective education for autism," Monitor on Psychology, vol. 35, no. 11, pp. 46-49, 2004.

[3] A. Landowska, A. Kołakowska, A. Anzulewicz, P. Jarmołkowicz, and J. Rewera, "E-technologie w diagnozie i pomiarach postepow terapii dzieci z autyzmem w Polsce," e-mentor, no. 4 (56), pp. 26-30, 2014.

[4] A. Landowska, A. Kolakowska, A. Anzulewicz, P. Jarmołkowicz, and J. Rewera, "E-technologie w edukacji i terapii dzieci z autyzmem w Polsce," EduAkcja. Magazyn edukacji elektronicznej, vol. 8, no. 2, pp. 42-48, 2014.

[5] A. Landowska and M. Smiatacz, "Mobile activity plan applications for behavioral therapy of autistic children," in Man-Machine Interactions 4. Springer, 2016, pp. 115-125.
[6] "Automated therapy monitoring for children with ASD, project website," http://autmon.eti.pg.gda.pl/, accessed: 2016-05-08.

[7] W. Jones and A. Klin, "Attention to eyes is present but in decline in 2-6-month-old infants later diagnosed with autism," Nature, vol. 504 no. 7480 , pp. $427-431$, 2013. doi: 10.1038 /nature 12715

[8] J. Hashemi, T. V. Spina, M. Tepper, A. Esler, V. Morellas, N. Papanikolopoulos, and G. Sapiro, "Computer vision tools for the noninvasive assessment of autism-related behavioral markers," IEEE International Conference on Development and Learning and Epigenetic Robotics, 2012. doi: 10.1109/devlrn.2012.6400865

[9] E. B. Torres, M. Brincker, R. W. Isenhower, P. Yanovich, K. A. Stigler, J. I. Nurnberger, D. N. Metaxas, and J. V. José, "Autism: the micromovement perspective." Frontiers in Integrative Neuroscience, vol. 7 2013. doi: 10.3389/fnint.2013.00032

[10] D. Wall, J. Kosmicki, T. Deluca, E. Harstad, and V. Fusaro, "Use of machine learning to shorten observation-based screening and diagnosis of autism," Translational psychiatry, vol. 2, no. 4, p. e100, 2012. doi: 10.1038/tp.2012.10

[11] D. Deriso, J. Susskind, L. Krieger, and M. Bartlett, "Emotion mirror: a novel intervention for autism based on real-time expression recognition," in Computer Vision-ECCV 2012. Workshops and Demonstrations. Springer, 2012, pp. 671-674. doi: 10.1007/978-3-642-33885-4_79

[12] R. El Kaliouby and P. Robinson, "The emotional hearing aid: an assistive tool for children with asperger syndrome," Universal Access in the Information Society, vol. 4, no. 2, pp. 121-134, 2005. doi 10.1007/s10209-005-0119-0

[13] A. Landowska, K. Karpienko, M. Wróbel, and M. JedrzejewskaSzczerska, "Selection of physiological parameters for optoelectronic system supporting behavioral therapy of autistic children," in Society of Photo-Optical Instrumentation Engineers (SPIE) Conference Series, vol. 9290, 2014. doi: 10.1117/12.2075020

[14] M. Jedrzejewska-Szczerska, K. Karpienko, and A. Landowska, "System supporting behavioral therapy for children with autism," Journal of Innovative Optical Health Sciences, vol. 8, no. 03, p. 1541008, 2015. doi: $10.1142 / \mathrm{s} 1793545815410084$

[15] J. C. Read, S. MacFarlane, and C. Casey, "Endurability, engagement and expectations: Measuring children's fun," in Interaction design and children, vol. 2. Shaker Publishing Eindhoven, 2002, pp. 1-23.

[16] G. Sim, S. MacFarlane, and J. Read, "All work and no play: Measuring fun, usability, and learning in software for children," Computers \& Education, vol. 46, no. 3, pp. 235-248, 2006. 